\title{
Analysis of EGNOS-Augmented GPS Receiver Positioning Accuracy
}

\author{
A. SKORKOWSKI* AND T. TOPÓR-KAMIŃSKI \\ Institute of Measurement Science, Electronics and Control, Silesian University of Technology \\ Akademicka 10, 44-100 Gliwice, Poland \\ This study presents the operation basis of GPS global positioning system and augmentation systems used to \\ increase positioning accuracy. Furthermore, selected measurement results for an EGNOS-augmented GPS receiver \\ positioning are included along with an analysis of GIS measurements accuracy, performed using differential \\ corrections. This analysis takes into consideration the impact of the number of measurement series and satellite \\ constellation on the accuracy of positioning achieved in praxis using the EGNOS system.
}

PACS: 43.10.Pr, 89.20.Hh, 06.20.Dk, 07.05.Hd

\section{Introduction}

Global Positioning System (GPS) is an example of a distributed measurement system constructed using the most advanced technology [1-4]. GPS receiver positioning accuracy is a product of many factors which will be discussed below. Methods of improving the GPS positioning accuracy along with selected study results of positioning accuracy achieved in praxis using the EGNOS system will be also demonstrated.

A GPS space segment is composed of at least 24 satellites travelling along 6 orbits -4 satellites in each orbit. The current constellation status of all GPS satellites (along with spare satellites) is registered by e.g. United States Naval Observatory. The satellites are located in orbits $20,200 \mathrm{~km}(12,600 \mathrm{mi})$ above the Earth surface and rotate around the planet twice a day. 5 satellites are within line of sight from everywhere on Earth's surface with the probability of $99.96 \%$.

The GPS control segment receives signals from the satellites and, based on that information, computes the corrections for their positions (ephemerides). The corrections are sent back to the satellites. The segment consists of the Master Control Station in Colorado Springs, 4 Monitor Stations in Hawaii, Ascension Island, Kwajalein Atoll, Diego Garcia Island, as well as 6 National Geospatial Agency stations in Argentina, Bahrain, Australia, Ecuador, the Great Britain and the USA.

There are two versions of the GPS system:

- PPS (Precise Positioning System) is available to the U.S. and NATO military as well as selected organizations for a fee;

- SPS (Standard Positioning System) is free of charge and commonly available (receiving the satellite signal is toll-free).

In order to use SPS it is necessary to have a GPS receiver which computes the location, speed and ac-

\footnotetext{
* corresponding author; e-mail: artur.skorkowski@polsl.pl
}

curate time on the basis of the data sent from the satellites. Most receivers manufactured nowadays are equipped with digital maps and software for processing data for the purposes of naval, air, automobile and even pedestrian navigations as well as geodesy. Information necessary to determine the position of GPS receiver is sent unidirectionally, i.e. the receiver is passive and does not send out any signals to the satellites. The satellites, on the other hand, send two types of signals separated by means of CDMA technology:

- $\mathrm{C} / \mathrm{A}$ on $\mathrm{L} 1$ carrier $=1575.42 \mathrm{MHz}(1.023 \mathrm{MHz}$ spectrum) received by all GPS devices (SPS and PPS),

- $\mathrm{P}$ on L2 carrier $=1227.60 \mathrm{MHz}(10.23 \mathrm{MHz}$ spectrum) received only by PPS devices.

Signals from four satellites are enough to determine the position of an object in three-dimensional space and calculate the accurate time. The signals include information about the satellite they come from and the time of the broadcast. The corrections of the current position provided by the control segment are also included. Using the information, the software embedded in the GPS receiver can establish: the real positions of the satellites at a particular moment in time and the distance between the receiver and the satellite, based on the satellite-receiver signal transmission time, thus making it possible to determine the position of the GPS receiver itself. The positioning computational algorithm is based on the technique of trilateration, a method of triangulation that, in brief, consists in calculating the distance between the GPS receiver and the three satellites, which in turn enables a precise determination of the position in space. For the GPS receiver to operate accurately it needs to sync with the satellites' clocks (accurate time determination). Therefore, it is necessary to receive data from four, not three, satellites (determining four unknowns - the spatial coordinates and the receiver's error in determining the time). 


\section{Positioning accuracy}

The receiver's error in determining the position and time is first and foremost a product of the following factors $[5,6]$ :

- ionospheric delay - changes in the speed of signals broadcast from the satellites and travelling through the ionosphere $( \pm 7 \mathrm{~m})$,

- tropospheric delay - analogous phenomenon in the troposphere caused by changes in air humidity, temperature and pressure $( \pm 0.5 \mathrm{~m})$,

- ephemerides error - differences between theoretical and actual position of the satellites $( \pm 2.5 \mathrm{~m})$,

- satellite clock inaccuracy $( \pm 2 \mathrm{~m})$,

- reception of reflected signals which reach the receiver by other routes than directly from the satellite $( \pm 1 \mathrm{~m})$,

- receiver errors - noise distorting the transmission, inaccuracy of computational procedures in the software $( \pm 1 \mathrm{~m})$.

PPS receivers are additionally provided with $\mathrm{P}$ signal on frequency L2 $=1227.60 \mathrm{MHz}$, which makes it possible to calculate a correction and neutralize the main cause of the measurement error resulting from the distortion of radio signals in ionosphere $[7,8]$.

Currently manufactured GPS receivers track a greater number of satellites (up to 12). If there are no distortions, the measurement error does not exceed $15 \mathrm{~m}$. Manufactured are also GPS receivers which make use of corrections from WAAS or EGNOS differential systems augmenting accuracy of positioning up to $1-5 \mathrm{~m}$.

\section{Differential corrections}

The GPS accuracy described above is not sufficient for many uses, such as paramedics, geodesic measurements or landing aircraft navigation. In situations where higher measurement accuracy is required, DPGS is used. It takes advantage of differential corrections in addition to the typical GPS data. This system takes advantage of the fact that positioning inaccuracy for civilian use is usually caused by the same set of factors in a small geographic area. There are two basic techniques of correcting errors in GPS receiver positioning: real-time correction and post-processing [9].

When GPS signal is received in a known and fixed location, it is possible to calculate the measurement inaccuracy and send that to the receivers located in the nearby area. This is how differential (referential) GPS stations operate. They receive signals from Navstar satellites, calculate the measurement error and send a correction to GPS receivers present in the nearby area that are able to receive such corrections.
It is possible to conduct a series of measurements without differential corrections and process them later using the data from a differential station. This technique is called post-processing and is very popular in geodesic measurements. In case of long measurements which simultaneously utilize at least two GPS receivers and differential corrections, it is possible to locate a geodesic point with $1 \mathrm{~cm}$ or even $1 \mathrm{~mm}$ accuracy.

A project of creating a civilian network of navigational satellites has emerged a few years ago because:

- there is a need to create a system which is more accurate than the existing ones and can be used worldwide (e.g. in air navigation and during rescue missions),

- a system which is independent from one country and its policies is more beneficial,

- a system with higher resistance to noise and satellite defects is required for many uses.

A concept of such a system has been proposed by the European Union. Initially the entire project was known by a name of Global Navigation Satellite System (GNSS). For economic reasons a system called EGNOS has been created first with a purpose of checking and correcting the data coming from Navstar network (and possibly Glonass). At the same time the Galileo system is being developed.

European Geostationary Navigation Overlay Service (EGNOS) is a European satellite based augmentation system (SBAS) which supports GPS and Glonass, as well as the future Galileo, built by the European Space Agency, the European Commission and EUROCONTROL. The main task of this system is to transmit differential corrections and provide information about GPS failures. The key goal of the system is to increase the accuracy and reliability of GPS positioning which will especially benefit air transport. An EGNOS counterpart in the North America is Wide Area Augmentation System (WAAS), in India - GAGAN (GPS-Aided Geosynchronous Augmented Navigation System - to be launched in 2013), and in Japan — MSAS (Multi-functional Satellite Augmentation System).

Geostationary satellites can be used as differential stations, too, in order to correct the data from the GPS network. EGNOS is based on three such satellites, which not only send out position and time corrections from GPS receivers but also inform about possible blackouts and failures of Navstar and Glonass systems. A similar system called WASS in USA consists of both geostationary satellites and ground referential stations. Differential station networks are built in e.g. Germany (SAPOS), Sweden (SWEPOS), Japan (MSAS with a geostationary satellite) and many other countries. In Poland there are referential station networks in Silesia and in Warsaw area with a range of $25 \mathrm{~km}(16 \mathrm{mi})$. There are also stations in Dziwnów and Rozewie (range of approximately $100 \mathrm{~km}$ ) 
which are used for navigational purposes mostly by naval entities.

The EGNOS space segment is composed of three geostationary satellites covering the entire Europe. On the ground there are measurement and control stations which constantly test the Navstar network and EGNOS satellites. GPS data corrections along with the data about the GPS network status are transmitted to EGNOS satellites which in turn broadcast them to the GPS receivers that are able to interpret them. One of EGNOS control stations is situated in the Space Research Centre in Warsaw.

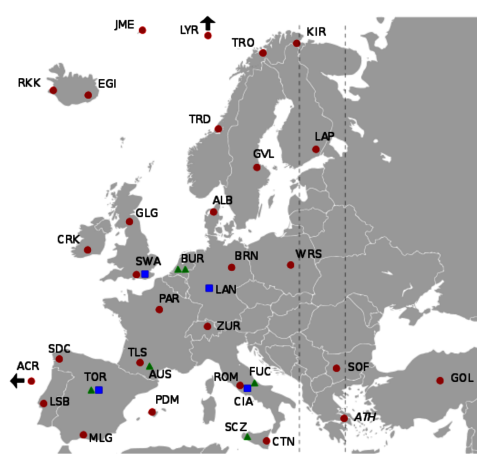

Fig. 1. Deployment of EGNOS ground stations $(\bullet-$ RIMS, - MCC, $\boldsymbol{\Delta}$ - NLES $)$.

The ground segment is composed of a series of stations (Fig. 1):

- 34 ranging and integrity monitoring stations (RIMS) which receive navigational dispatches from GPS satellites; one such station is located in Warsaw;

- 4 mission control centres (MCCs) which process data and compute differential corrections (in Poland there in one station of that kind in Warsaw),

- 6 navigation land earth station (NLES) which send out corrections to satellites that subsequently transmit them to the users,

- 2 control and test stations: Development Verification Platform (DVP) and Application Specific Qualification Facility (ASQF) in Torrejón near Madrid, Spain and Performance Assessment and Check-out Facility (PACF) in Toulouse, France.

First of all, EGNOS checks data coming from the Navstar network, ensuring that there have not been any satellite defects transmission errors. Thanks to this, data from Navstar/EGNOS can be used in cases high accuracy information is needed for safety considerations. These are so called "Safety of Life" applications, e.g. precise aircraft navigation, train traffic management or some rescue missions.

Theoretically speaking, EGNOS should augment the positioning accuracy up to 3-5 meters. Unfortunately, research conducted in PAN Space Research Centre have demonstrated that in Poland this accuracy is lower as the system does not transmit corrections for satellites which are within line of sight in Poland. Increasing the number of satellites transmitting EGNOS signal is supposed to improve the situation. They will be deployed in geostationary orbits located over $5^{\circ} \mathrm{E}$ and $31.5^{\circ} \mathrm{E}$ meridians.

\section{Study results}

In this section examples of measurements will be demonstrated which were made with GPS Hi-Target Qmini M1 receiver in a location typical for Geographic Information Systems (GIS) measurements, i.e. partially covered (the blocking object's height - approximately $8 \mathrm{~m}$, the distance from the measurement point - approximately $6 \mathrm{~m}$ ). In order to increase accuracy, measurements of this kind are conducted at present as differential measurements with the help of corrections received from appropriate satellites or a GPRS network.

GIS measurements are usually carried out statically and repeatedly (in measurement series) and complemented by attributes that describe the measurement spot. Figure 2 shows how number of measurement series $(n)$ impacts dispersion of received measurement results

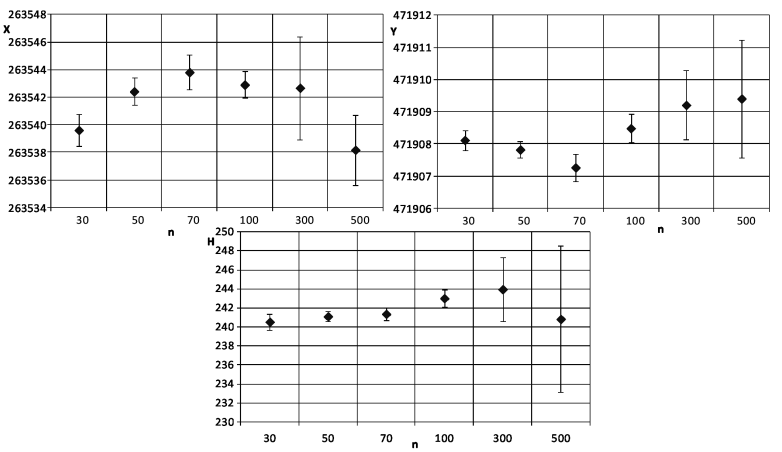

Fig. 2. Relations between average coordinates of $X$, $Y, H$ positions along with standard deviations of these averages from the number $n$ of measurement series.

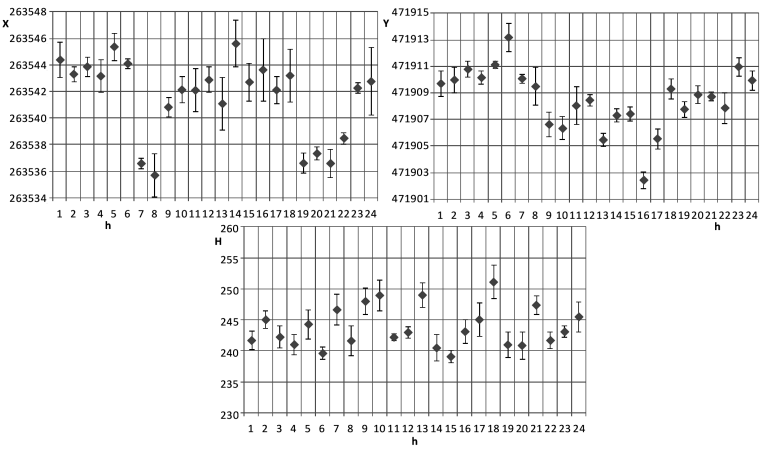

Fig. 3. Relations between average coordinates of $X, Y$, $H$ positions and standard deviations of these averages (series with $n=100$ ) from the time (h) of measurements. 
which are set in the Cartesian coordinate system. Series with a number $n<30$ have also been analyzed but turned out to be unrepresentative.

The next step in the analysis was checking whether the satellite constellation influenced the results and the accuracy during measurements. In order to analyze all possible constellations, the measurements were conducted at different times of the day and night. Selected results received each hour are shown in Fig. 3. Measurements were conducted in $n=100$ series. Usually all measurement results in a series were received with a SBAS correction but justifiably results were added, which had a differential DGPS character only partially due to limitations in EGNOS availability. Measurements done in the evening and in the morning were partially autonomic measure- ments, i.e. not all results in the series had corrections. An effect of such type of measurements is a higher systematic error, which can be observed in Fig. 3 for the measurements done at 7-8 a.m. and 7-10 p.m.

If in the series only a small number of measurements are autonomic GPS measurements and the majority are DGPS differential measurements, the impact on the results dispersion is not significant (average standard deviation).

Autonomic GPS measurements in comparison with DGPS differential measurements, augmented by e.g. EGNOS, are characterized by a significantly lower accuracy and greater systematic errors, which is demonstrated by results shown in Table.

Average values of coordinates of $X, Y, H$ positions and standard deviations of these averages $U_{X}, U_{Y}, U_{H}$

TABLE for differential DGPS measurements and autonomic GPS measurements conducted in $n=100$ series.

\begin{tabular}{c|c|c|c|c|c|c|c|c|c|c|c|c}
\hline \hline & $X_{\min }$ & $X_{\max }$ & $X$ & $Y_{\min }$ & $Y_{\max }$ & $Y$ & $H_{\min }$ & $H_{\max }$ & $H$ & $U_{X}$ & $U_{Y}$ & $U_{H}$ \\
\hline DGPS & 263541.14 & 263545.02 & 263543.18 & 471909.14 & 471911.18 & 471910.18 & 238.01 & 244.01 & 241.06 & 1.201 & 0.510 & 1.672 \\
GPS & 263526.61 & 263540.95 & 263535.75 & 471907.58 & 471923.91 & 471911.92 & 232.71 & 254.31 & 244.79 & 3.101 & 3.832 & 5.401 \\
\hline
\end{tabular}

\section{Conclusions}

In the study analyses of GIS measurements accuracy, performed using differential corrections have been conducted. These analyses take into consideration the influence of the number of measurement series and satellite constellation on the accuracy of positioning achieved in praxis using the EGNOS system.

Autonomic GPS measurement makes it possible to determine the position of a receiver with an accuracy of a few or a dozen meters, depending on the number of the observed satellites and their geometrical distribution (constellation). The results of the static measurements of GPS receiver position shown in the study and taking into consideration differential correction for EGNOS system explicitly indicate that the accuracy of positioning is increased up to the level of a few meters, regardless of satellite constellation.

Because of the popularization of simple receivers which work with WAAS or EGNOS corrections, SBAS augmentation systems seem to constitute a convenient alternative to the technique of post-processing for the purpose of augmentation of GIS measurement accuracy.

It can be suspected that the increase in reliability, availability and correctness of information acquired from EGNOS will also make it possible to use it for "Safety of Life" applications, e.g. precise aircraft navigation, train traffic management or some rescue missions. Reaching this goal is expensive, though, as it requires an increase in the number of satellites broadcasting EGNOS signal as well as construction of new RIMS and MCC stations.

\section{References}

[1] B. Hofmann-Vellenhof, H. Lichtenegger, J. Collins, Global Positioning System (GPS) Theory and Practice, Springer, Vien 2001.

[2] J. Narkiewicz, Global Positioning GPS System Construction, Acting and Application, WKL, Warsaw 2003 (in Polish).

[3] K. Gut, J. Phys. IV: JP (France) 129, 109 (2005).

[4] W. Nawrocki, Dispersion Measuring Systems, WKL, Warsaw 2006 (in Polish).

[5] K. Barczak, T. Pustelny, Z. Zycki, T. Plazejczak, Acta Phys. Pol. A 116, 250 (2009).

[6] C. Specht, System GPS, Bernardinum, Pelplin (Poland) 2007.

[7] J. Ventura-Traveset, D. Flament, EGNOS, the European Geostationary Navigation Overlay System Cornerstone of Galileo, ESA, Noordwijk (Norway) 2006.

[8] T. Topór-Kaminski, R. Żurkowski, M. Grygiel, Acta Phys. Pol. A 120, 748 (2011).

[9] J. Lamparski, K. Swiatek, GPS in Geodesic Practice GAll, Katowice (Poland) 2007 (in Polish). 